\title{
INEQUALITIES CONCERNING ULTRASPHERICAL POLYNOMIALS AND BESSEL FUNCTIONS
}

\section{OTTO SZÁSZ ${ }^{1}$}

1. Introduction. Ultraspherical polynomials are defined by the recurrence formula

(1.1) $(n+1) P_{n+1}^{\lambda}(x)=2(n+\lambda) x P_{n}^{\lambda}(x)-(n+2 \lambda-1) P_{n-1}^{\lambda}(x)$, with $P_{0}^{\lambda}=1, P_{1}^{\lambda}=2 \lambda x$. Thus we have

$$
2 P_{2}^{\lambda}=2 \lambda\left[2(1+\lambda) x^{2}-1\right],
$$

and so on.

For $\lambda=1 / 2$ we get the Legendre polynomials $P_{n}(x)$. They satisfy the following interesting inequality:

$$
\begin{aligned}
\Delta_{n}(x) \equiv & {\left[P_{n}(x)\right]^{2} } \\
& -\left[P_{n-1}(x)\right]\left[P_{n+1}(x)\right] \geqq 0, \quad n \geqq 1,-1 \leqq x \leqq 1,
\end{aligned}
$$

with equality for $x= \pm 1$. This result is due to $P$. Turán, as stated in a recent paper by G. Szegö, ${ }^{2}$ where four elegant proofs of the theorem are given. The first proof (similar to that of Turán) is based on Mehler's formula, while the third proof is based on the identity

$$
\sum_{n=0}^{\infty} \frac{P_{n}(x)}{n !} z^{n}=e^{x_{z}} J_{0}\left[\left(1-x^{2}\right)^{1 / 2} z\right],
$$

and on the fact that the Bessel function $J_{0}$ has all its roots real.

Szegö remarks that the formula

$$
\sum_{n=0}^{\infty} \frac{P_{n}^{\lambda}(x)}{P_{n}^{\lambda}(1)} \cdot \frac{z^{n}}{n !} \text {. }
$$

$=2^{\lambda-1 / 2} \Gamma(\lambda+1 / 2) e^{x z}\left[\left(1-x^{2}\right)^{1 / 2} z\right]^{1 / 2-\lambda} J_{\lambda-1 / 2}\left[\left(1-x^{2}\right)^{1 / 2} z\right], \lambda>-1 / 2$,

and analogous formulas for Laguerre and Hermite polynomials yield inequalities analogous to (1.2) for ultraspherical, Laguerre, and 1948.

Presented to the Society, September 7, 1948; received by the editors December 28,

1 This paper was written at the Institute for Numerical Analysis of the National Bureau of Standards, with the financial support of the Office of Naval Research of the U.S. Navy Department.

2 G. Szegö, On an inequality of P. Turan concerning Legendre polynomials, Bull. Amer. Math. Soc. vol. 54 (1948) pp. 401-405. 
Hermite polynomials. Thus

$$
\left[\frac{P_{n}^{\lambda}(x)}{P_{n}^{\lambda}(1)}\right]^{2}-\frac{P_{n-1}^{\lambda}(x) P_{n+1}^{\lambda}(x)}{P_{n-1}^{\lambda}(1) P_{n+1}^{\lambda}(1)} \geqq 0, \quad \lambda>-1 / 2 .
$$

We have, ${ }^{3}$ for $\lambda>-1 / 2$,

$$
P_{n}^{\lambda}(1)=C_{n+2 \lambda-1, n}=\frac{2 \lambda \Gamma(n+2 \lambda)}{n ! \Gamma(2 \lambda+1)}, \quad n=0,1, \cdots .
$$

so that (1.3) can be restated as

$$
n(n+2 \lambda)\left[P_{n}^{\lambda}(x)\right]^{2} \geqq(n+1)(n+2 \lambda-1) P_{n-1}^{\lambda}(x) P_{n+1}^{\lambda}(x) ;
$$

with $\lambda=1 / 2$, this reduces to the inequality (1.2).

We write $F_{n}(x)$ for $P_{n}^{\lambda}(x) / P_{n}^{\lambda}(1)$; then (1.3) becomes $\Delta_{n}^{\lambda}(x) \equiv\left[F_{n}(x)\right]^{2}$ $-F_{n-1}(x) F_{n+1}(x) \geqq 0$, and equality holds for $x=1$. We have

$$
F_{0}(x) \equiv 1, \quad F_{1}(x)=x, \quad F_{2}(x)=\frac{2(1+\lambda) x^{2}-1}{1+2 \lambda}, \ldots .
$$

For $0<\lambda<1$ we derive a sharper inequality, employing essentially the recursion formula (1.1). The result is

$$
\frac{\lambda\left[1-F_{n}^{2}(x)\right]}{(n+\lambda-1)(n+2 \lambda)} \leqq \Delta_{n}^{\lambda}(x)<\frac{2 \lambda \Gamma(2 \lambda)}{\lambda+1} \frac{\Gamma(n)}{\Gamma(n+2 \lambda)},
$$

$-1 \leqq x \leqq 1$. In particular, for $\lambda=1 / 2$,

$$
\frac{1-\left[P_{n}(x)\right]^{2}}{(2 n-1)(n+1)} \leqq \Delta_{n}(x)<\frac{2 n+1}{3 n(n+1)} ;
$$

the smallness of $\Delta_{n}$ indicates that the inequality (1.2) is rather deep.

For Bessel functions we shall establish the inequality

$$
J_{\mu}^{2}(t)-J_{\mu-1}(t) J_{\mu+1}(t)>\frac{1}{\mu+1} J_{\mu}^{2}(t), \quad \mu>0, t \text { real } .
$$

It seems particularly interesting that the procedure is very much the same in deriving (1.5) and (1.6), and is based mainly on the respective recurrence relations.

2. Ultraspherical polynomials; $\lambda<1$. From (1.1) and (1.4), we obtain

${ }^{3}$ G. Szegö, Orthogonal polynomials, Amer. Math. Soc. Colloquium Publications, vol. 23,1939 , pp. 80,166 . Referred to as O. P. 


$$
\begin{aligned}
(n+1) F_{n+1}(x)= & 2(n+\lambda) x F_{n}(x) \frac{\Gamma(n+2 \lambda)(n+1)}{\Gamma(n+1+2 \lambda)} \\
& -(n+2 \lambda-1) F_{n-1}(x) \frac{\Gamma(n+2 \lambda-1) n(n+1)}{\Gamma(n+1+2 \lambda)},
\end{aligned}
$$

or

$$
F_{n+1}(x)=\frac{2(n+\lambda) x}{n+2 \lambda} F_{n}(x)-\frac{n}{n+2 \lambda} F_{n-1}(x), \quad n \geqq 1 .
$$

It follows that

(2.2) $\quad F_{n+1}(x) F_{n}(x)=\frac{2(n+\lambda)}{n+2 \lambda} x F_{n}^{2}(x)-\frac{n}{n+2 \lambda} F_{n-1}(x) F_{n}(x)$.

From (2.1) we get

$$
F_{n}(x)=\frac{2(n+\lambda-1) x}{n+2 \lambda-1} F_{n-1}(x)-\frac{(n-1)}{n+2 \lambda-1} F_{n-2}(x), \quad n \geqq 2,
$$

whence substitution into (2.2) yields

$$
\begin{aligned}
& \frac{2 x(n+\lambda)}{n+2 \lambda} F_{n}^{2}(x) \\
& =F_{n+1}(x)\left\{\frac{2(n+\lambda-1)}{n+2 \lambda-1} x F_{n-1}(x)-\frac{n-1}{n+2 \lambda-1} F_{n-2}(x)\right\} \\
& +\frac{n}{n+2 \lambda} F_{n-1}(x)\left\{\frac{2(n+\lambda-1)}{n+2 \lambda-1} x F_{n-1}(x)-\frac{n-1}{n+2 \lambda-1} F_{n-2}(x)\right\} \\
& =\frac{2 n x(n+\lambda-1)}{(n+2 \lambda-1)(n+2 \lambda)} F_{n-1}^{2}(x)+F_{n+1}(x) \frac{2(n+\lambda-1)}{n+2 \lambda-1} x F_{n-1}(x) \\
& -\frac{(n-1)}{n+2 \lambda-1} F_{n-2}(x)\left\{\frac{2(n+\lambda) x}{n+2 \lambda} F_{n}(x)-\frac{n}{n+2 \lambda} F_{n-1}(x)\right\} \\
& -\frac{(n-1) n}{(n+2 \lambda-1)(n+2 \lambda)} F_{n-2}(x) F_{n-1}(x) \\
& =\frac{2 n(n+\lambda-1) x}{(n+2 \lambda-1)(n+2 \lambda)} F_{n-1}^{2}(x)+2 x \frac{n+\lambda-1}{n+2 \lambda-1} F_{n-1}(x) F_{n+1}(x) \\
& -\frac{2(n-1)(n+\lambda) x}{(n+2 \lambda-1)(n+2 \lambda)} F_{n-2}(x) F_{n}(x), \quad n \geqq 2 .
\end{aligned}
$$

If we write 


$$
\begin{aligned}
(n+\lambda)(n+2 \lambda & -1) F_{n}^{2}(x) \\
& -(n+\lambda-1)(n+2 \lambda) F_{n-1}(x) F_{n+1}(x)=D_{n}^{\lambda}(x)
\end{aligned}
$$

then

$$
\begin{aligned}
D_{n}^{\lambda}(x)= & n(n+\lambda-1) F_{n-1}^{2}(x)-(n-1)(n+\lambda) F_{n-2}(x) F_{n}(x) \\
= & \left\{(n+\lambda-1)(n+2 \lambda-2) F_{n-1}^{2}(x)\right. \\
& \left.-(n+\lambda-2)(n+2 \lambda-1) F_{n-2}(x) F_{n}(x)\right\} \\
& \cdot \frac{(n-1)(n+\lambda)}{(n+\lambda-2)(n+2 \lambda-1)}+F_{n-1}^{2}(x) \\
& \cdot\left\{n(n+\lambda-1)-\frac{(n+\lambda-1)(n+2 \lambda-2)(n-1)(n+\lambda)}{(n+\lambda-2)(n+2 \lambda-1)}\right\} \\
= & D_{n-1}^{\lambda}(x) \frac{(n-1)(n+\lambda)}{(n+\lambda-2)(n+2 \lambda-1)}+(n+\lambda-1) F_{n-1}^{2}(x) \\
& \cdot \frac{n(n+\lambda-2)(n+2 \lambda-1)-(n-1)(n+\lambda)(n+2 \lambda-2)}{(n+\lambda-2)(n+2 \lambda-1)} \\
= & \frac{(n-1)(n+\lambda)}{(n+\lambda-2)(n+2 \lambda-1)} D_{n-1}^{\lambda}(x) \\
& +\frac{2 \lambda(\lambda-1)(n+\lambda-1) F_{n-1}^{2}(x)}{(n+\lambda-2)(n+2 \lambda-1)} .
\end{aligned}
$$

Let

$$
g_{n}=g_{n-1} \frac{(n+\lambda-2)(n+2 \lambda-1)}{(n-1)(n+\lambda)}, \quad n \geqq 2, g_{1}=1 \text {; }
$$

then

$$
g_{n} D_{n}^{\lambda}(x)=g_{n-1} D_{n-1}^{\lambda}(x)+2 \lambda(\lambda-1) \frac{n+\lambda-1}{(n-1)(n+\lambda)} g_{n-1} F_{n-1}^{2}(x),
$$

where, from (2.3),

$$
\begin{aligned}
D_{1}^{\lambda}(x) & =2 \lambda(1+\lambda) F_{1}^{2}(x)-\lambda(1+2 \lambda) F_{0} F_{2}(x) \\
& =\lambda\left\{2(1+\lambda) x^{2}-2(1+\lambda) x^{2}+1\right\} \\
& =\lambda .
\end{aligned}
$$

From (2.4), we have 


$$
\begin{aligned}
g_{n} & =\prod_{2}^{n} \frac{(\nu+\lambda-2)(\nu+2 \lambda-1)}{(\nu-1)(\nu+\lambda)}=\frac{\lambda(\lambda+1) \prod_{2}^{n}(\nu+2 \lambda-1)}{(n-1) !(n+\lambda-1)(n+\lambda)} \\
& =\frac{\lambda(\lambda+1)}{(n+\lambda-1)(n+\lambda)} \prod_{1}^{n-1}\left(1+\frac{2 \lambda}{\nu}\right)=O\left(n^{2 \lambda-2}\right),
\end{aligned}
$$

as $n \rightarrow \infty$.

It is well known [cf. O. P. pp. 80, 166] that, for $\lambda>0$,

$$
\max _{-1 \leqq x \leqq 1}\left|P_{n}^{\lambda}(x)\right|=P_{n}^{\lambda}(1)=C_{n+2 \lambda-1, n},
$$

so that

$$
\left|F_{n}(x)\right| \leqq F_{n}(1)=1
$$

Hence

$$
\frac{n+\lambda}{n(n+\lambda+1)} g_{n} F_{n}^{2}(x) \leqq \frac{g_{n}}{n}=O\left(n^{2 \lambda-3}\right) .
$$

We now consider the case $0<\lambda<1$; it follows from (2.9) that the series

$$
\sum_{2}^{\infty}\left\{g_{n-1} D_{n-1}^{\lambda}(x)-g_{n} D_{n}^{\lambda}(x)\right\}
$$

is uniformly convergent in $-1 \leqq x \leqq 1$, and from (2.5), (2.6) we have

$$
\lambda-\lim _{n \rightarrow \infty} g_{n} D_{n}^{\lambda}(x)=2 \lambda(1-\lambda) \sum_{1}^{\infty} \frac{(n+\lambda) g_{n}}{n(n+\lambda+1)} F_{n}^{2}(x) .
$$

We write

$$
\begin{aligned}
\lim _{n \rightarrow \infty} g_{n} D_{n}^{\lambda}(x) & =\lambda\left\{1-2(1-\lambda) \sum_{1}^{\infty} \frac{(n+\lambda) g_{n}}{n(n+\lambda+1)} F_{n}^{2}(x)\right\} \\
& \equiv \chi(x),
\end{aligned}
$$

a continuous function of $x$ in $-1 \leqq x \leqq 1$. It follows from (2.8) and (2.10) that

$$
\chi(x) \geqq \chi(1)=\lambda\left\{1-2(1-\lambda) \sum_{1}^{\infty} \frac{(n+\lambda) g_{n}}{n(n+\lambda+1)}\right\} .
$$

We next show that 


$$
\chi(1)=\lim g_{n} D_{n}^{\lambda}(1)=0 .
$$

From (2.3), we have

$$
D_{n}^{\lambda}(1)=(n+\lambda)(n+2 \lambda-1)-(n+\lambda-1)(n+2 \lambda)=\lambda,
$$

and from (2.7), for $0<\lambda<1$, we see that $g_{n} \rightarrow 0$; this proves (2.12).

It now follows from (2.11) that

$$
\chi(x) \geqq 0=1-2(1-\lambda) \sum_{1}^{\infty} \frac{(n+\lambda) g_{n}}{n(n+\lambda+1)},
$$

so that

$$
\sum_{1}^{\infty} \frac{(n+\lambda) g_{n}}{n(n+\lambda+1)}=\frac{1}{2(1-\lambda)}, \quad 0<\lambda<1 .
$$

Note that by (2.7) we have

$$
g_{n}=\frac{\lambda(\lambda+1) \Gamma(n+2 \lambda)}{\Gamma(1+2 \lambda)(n+\lambda-1)(n+\lambda) \Gamma(n)} \sim \frac{\lambda(\lambda+1)}{\Gamma(1+2 \lambda)} n^{2 \lambda-2} .
$$

From (2.5) and (2.6) we get

$$
g_{n} D_{n}^{\lambda}(x)=\lambda-2 \lambda(1-\lambda) \sum_{\nu=1}^{n-1} \frac{g_{\nu}(\nu+\lambda) F_{\nu}^{2}(x)}{\nu(\nu+\lambda+1)} ;
$$

furthermore

$$
g_{n} D_{n}^{\lambda}(x)=\chi(x)+2 \lambda(1-\lambda) \sum_{n}^{\infty} \frac{g_{\nu}(\nu+\lambda)}{\nu(\nu+\lambda+1)} F_{\nu}^{2}(x) .
$$

Now from (2.8), (2.12), and (2.13) it follows that

$$
\begin{aligned}
g_{n} D_{n}^{\lambda}(x)= & 2 \lambda(1-\lambda) \sum_{1}^{n-1} \frac{g_{\nu}(\nu+\lambda)}{\nu(\nu+\lambda+1)}\left\{1-F_{\nu}^{2}(x)\right\} \\
& +\sum_{n}^{\infty} \frac{g_{\nu}(\nu+\lambda)}{\nu(\nu+\lambda+1)} \geqq \sum_{n}^{\infty} \frac{g_{\nu}(\nu+\lambda)}{\nu(\nu+\lambda+1)} \\
= & g_{n} D_{n}^{\lambda}(1)=\lambda g_{n} .
\end{aligned}
$$

It follows from (2.14) that, uniformly in $-1 \leqq x \leqq 1$,

$$
g_{n} D_{n}^{\lambda}(x) \downarrow \chi(x),
$$

furthermore, 


$$
D_{n}^{\lambda}(x) \geqq D_{n}^{\lambda}(1)=\lambda .
$$

From (2.15) we have

$$
g_{n} D_{n}^{\lambda}(x)<g_{1} D_{1}^{\lambda}=\lambda .
$$

From (2.3), (2.13), and (2.16) we obtain

$$
\begin{aligned}
D_{n}^{\lambda}(x)= & (n+\lambda-1)(n+2 \lambda)\left\{F_{n}^{2}(x)-F_{n-1}(x) F_{n+1}(x)\right\} \\
& +\lambda F_{n}^{2}(x) \geqq \lambda,
\end{aligned}
$$

so that

$$
\Delta_{n}^{\lambda}(x)=F_{n}^{2}(x)-F_{n-1}(x) F_{n+1}(x) \geqq \frac{\lambda\left(1-F_{n}^{2}(x)\right)}{(n+\lambda-1)(n+2 \lambda)},
$$

and

$$
n^{2 \lambda} \Delta_{n}^{\lambda}(x) \rightarrow \frac{\Gamma(1+2 \lambda)}{\lambda(\lambda+1)} \chi(x),
$$

uniformly in $-1 \leqq x \leqq 1$.

Summarizing, we have the following theorem:

Theorem 1. Denote by $P_{n}^{\lambda}(x)$ the ultraspherical polynomial and let

$$
F_{n}(x)=\frac{P_{n}^{\lambda}(x)}{P_{n}^{\lambda}(1)}, \quad \Delta_{n}^{\lambda}(x)=F_{n}^{2}(x)-F_{n-1}(x) F_{n+1}(x) .
$$

If $0<\lambda<1$, then $n^{2 \lambda} \Delta_{n}^{\lambda}(x)$ tends uniformly to a non-negative function, and

$$
\begin{aligned}
& \frac{\lambda\left(1-F_{n}^{2}(x)\right)}{(n+\lambda-1)(n+2 \lambda)} \leqq \Delta_{n}^{\lambda}(x)<\frac{\Gamma(1+2 \lambda)}{\lambda+1} \cdot \frac{\Gamma(n)}{\Gamma(n+2 \lambda)}, \\
& -1 \leqq x \leqq 1 .
\end{aligned}
$$

In particular for $\lambda=1 / 2$ (the Legendre polynomial), we have

$$
\frac{1-\left(P_{n}(x)\right)^{2}}{(2 n-1)(n+1)} \leqq\left(P_{n}(x)\right)^{2}-P_{n-1}(x) P_{n+1}(x)<\frac{2 n+1}{3 n(n+1)} .
$$

3. The case $\lambda>1$. Let now $\lambda>1$; from (2.14) we have

$$
g_{n} D_{n}^{\lambda}(x)=\lambda+2 \lambda(\lambda-1) \sum_{1}^{n-1} \frac{g_{\nu}(\nu+\lambda)}{\nu(\nu+\lambda+1)} F_{\nu}^{2}(x),
$$


so that $g_{n} D_{n}^{\lambda}(x)$ increases as $n$ increases. Hence

$$
g_{n} D_{n}^{\lambda}(x) \geqq \lambda,
$$

or

$$
(n+\lambda-1)(n+2 \lambda) g_{n} \Delta_{n}^{\lambda}(x)+\lambda g_{n} F_{n}^{2}(x) \geqq \lambda=D_{n}^{\lambda}(1) .
$$

Furthermore

$$
g_{n} D_{n}^{\lambda}(x) \leqq g_{n} D_{n}^{\lambda}(1)=\lambda+2 \lambda(\lambda-1) \sum_{1}^{n-1} \frac{g_{v}(\nu+\lambda)}{\nu(\nu+\lambda+1)}=g_{n} \lambda .
$$

Thus

$$
\Delta_{n}^{\lambda}(x) \geqq \frac{\lambda}{(n+\lambda-1)(n+2 \lambda) g_{n}}-\frac{\lambda F_{n}^{2}(x)}{(n+\lambda-1)(n+2 \lambda)},
$$

and

$$
\Delta_{n}^{\lambda}(x) \leqq \frac{\lambda\left(1-F_{n}^{2}(x)\right)}{(n+\lambda-1)(n+2 \lambda)} .
$$

In this case, from (2.7), we have $g_{n} \uparrow \infty$, and

$$
\Delta_{n}^{\lambda}(x)=O\left(n^{-2}\right) \text {, }
$$

as $n \rightarrow \infty$.

Finally, for $\lambda=1$, we have

$$
\begin{gathered}
F_{n}(x)=\frac{\sin (n+1) \theta}{(n+1) \sin \theta}, \quad x=\cos \theta, \\
g_{n} D_{n}^{\lambda}(x)=g_{1} D_{1}^{\lambda}=1, \quad g_{n}=1, \quad D_{n}^{\lambda}(x)=1, \\
n(n+2) \Delta_{n}(x)=1-F_{n}^{2}(x) .
\end{gathered}
$$

4. An application. If $P_{n+1}(x)=0$, then from (2.17) we obtain

$$
\frac{1-P_{n}^{2}(x)}{(n+1)(2 n-1)} \leqq P_{n}^{2}(x)<\frac{2 n+1}{3 n(n+1)},
$$

or

$$
\frac{1}{n(2 n+1)} \leqq P_{n}^{2}(x)<\frac{2 n+1}{3 n(n+1)} .
$$

If $P_{n}(t)=0$, then 


$$
\frac{1}{(n(2 n+1))^{1 / 2}} \leqq\left|P_{n}(x)\right|=\left|\int_{t}^{x} P_{n}^{\prime}(u) d u\right|,
$$

which yields a lower bound for $[x-t]$. To this end we employ the formula [O. P. p. 83]

$$
\frac{d}{d x} P_{n}^{\lambda}(x)=2 \lambda P_{n-1}^{\lambda+1}(x)
$$

so that, for $\lambda=1 / 2$,

$$
P_{n}^{\prime}(x)=P_{n-1}^{3 / 2}(x) ;
$$

hence, from (1.5), for $\lambda=3 / 2$,

$$
\left|P_{n}^{\prime}(x)\right| \leqq C_{n+1, n-1}=(3 / 2) n(n+1) ;
$$

equality holds for $x= \pm 1$.

It follows that

$$
\frac{1}{(n(2 n+1))^{1 / 2}}<|x-t|(3 / 2) n(n+1),
$$

or

$$
|x-t|>2 / 3 n^{-3 / 2}(2 n+1)^{-1 / 2}(n+1)^{-1},
$$

where $P_{n+1}(x)=0, P_{n}(t)=0$.

5. Hermite polynomials. The situation is simpler for Hermite polynomials $H_{n}(x)$, defined by

$$
\begin{aligned}
H_{n+1}(x) & =2 x H_{n}(x)-2 n H_{n-1}(x), \quad n=1,2,3, \cdots, \\
H_{0} & =1, \quad H_{1}=2 x .
\end{aligned}
$$

In this case [see Amer. Math. Monthly vol. 55 (1948) solution of problem 4215, pp. 34-35],

$$
\frac{1}{2^{n+1} n !}\left\{H_{n+1}^{2}-H_{n} H_{n+2}\right\}=\sum_{v=0}^{n} \frac{1}{\nu ! 2^{\nu}} H_{\nu}^{2}
$$

hence monotone increasing, as $\boldsymbol{n}$ increases.

6. Bessel functions. The Bessel function of order $\mu$ is defined for $\mu>-1$ by the power series

$$
J_{\mu}(t)=(t / 2)^{\mu} \sum_{\nu=0}^{\infty} \frac{(-1)^{\nu}(t / 2)^{2 \nu}}{\nu ! \Gamma(\mu+\nu+1)} .
$$


It satisfies the recursion formula

$$
J_{\mu+1}(t)=\frac{2 \mu}{t} J_{\mu}(t)-J_{\mu-1}(t), \quad \quad \mu>0 .
$$

We write

$$
\Lambda_{\mu}(t)=(2 / t)^{\mu} \Gamma(\mu+1) J_{\mu}(t)=\Lambda_{\mu}(-t) .
$$

It then follows from (6.2) that

$$
\frac{t^{2} \Lambda_{\mu+1}(t)}{4 \mu(\mu+1)}=\Lambda_{\mu}(t)-\Lambda_{\mu-1}(t)
$$

and

$$
\begin{aligned}
\Lambda_{\mu}^{2}(t)= & \frac{t^{2}}{4 \mu(\mu+1)} \Lambda_{\mu}(t) \Lambda_{\mu+1}(t)+\Lambda_{\mu-1}(t) \Lambda_{\mu}(t) \\
= & \frac{t^{2}}{4 \mu(\mu+1)} \Lambda_{\mu+1}(t)\left\{\frac{4 \mu(\mu-1)}{t^{2}}\left(\dot{\Lambda}_{\mu-1}(t)-\Lambda_{\mu-2}(t)\right)\right\} \\
& +\Lambda_{\mu-1}(t) \Lambda_{\mu}(t) .
\end{aligned}
$$

Thus, using (6.4) again, we have

$$
\begin{aligned}
\Lambda_{\mu}^{2}= & \frac{\mu-1}{\mu+1}\left(\Lambda_{\mu+1} \Lambda_{\mu-1}-\Lambda_{\mu+1} \Lambda_{\mu-2}\right) \\
& +\frac{4 \mu(\mu-1)}{t^{2}} \Lambda_{\mu-1}\left(\Lambda_{\mu-1}-\Lambda_{\mu-2}\right),
\end{aligned}
$$

and

$$
\begin{aligned}
\Lambda_{\mu}^{2}-\frac{\mu-1}{\mu+1} \Lambda_{\mu-1} \Lambda_{\mu+1}= & \frac{4 \mu(\mu-1)}{t^{2}} \Lambda_{\mu-1}\left(\Lambda_{\mu-1}-\Lambda_{\mu-2}\right) \\
& -\frac{\mu-1}{\mu+1} \Lambda_{\mu-2}\left\{\Lambda_{\mu}-\Lambda_{\mu-1}\right\} \frac{4 \mu(\mu+1)}{t^{2}} \\
= & \frac{4 \mu(\mu-1)}{t^{2}} \Lambda_{\mu-1}^{2}-\frac{4 \mu(\mu-1)}{t^{2}} \Lambda_{\mu-2} \Lambda_{\mu .}
\end{aligned}
$$

If we put

$$
D_{\mu}(t)=\Lambda_{\mu}^{2}-\frac{\mu-1}{\mu+1} \Lambda_{\mu-1} \Lambda_{\mu+1}
$$

and 


$$
\Delta_{\mu}(t)=\Lambda_{\mu}^{2}-\Lambda_{\mu-1} \Lambda_{\mu+1}
$$

then we have

$$
D_{\mu}=\frac{4 \mu(\mu-1)}{t^{2}} \Delta_{\mu-1}
$$

and

$$
\frac{\mu-1}{\mu+1} \Delta_{\mu}=D_{\mu}-\frac{2}{\mu+1} \Lambda_{\mu}^{2} \text {. }
$$

Now from (6.5) we get

$$
D_{\mu}(t)=\frac{4 \mu^{2}(\mu-1)}{(\mu-2) t^{2}} D_{\mu-1}(t)-\frac{8 \mu(\mu-1)}{(\mu-2) t^{2}} \Lambda_{\mu-1}^{2} \quad(\mu \neq 2) ;
$$

hence

(6.7) $\frac{t^{2 \mu} D_{\mu}(t)}{4^{\mu}(\mu-1) \Gamma^{2}(\mu+1)}=\frac{t^{2(\mu-1)} D_{\mu-1}(t)}{4^{\mu-1}(\mu-2) \Gamma^{2}(\mu)}-\frac{2 t^{2(\mu-1)} \Lambda_{\mu-1}^{2}}{4^{\mu-1} \mu(\mu-2) \Gamma^{2}(\mu)}$.

It follows that the sequence

$$
Q_{\mu+\nu}(t)=\frac{t^{2}(\mu+\nu) D_{\mu+\nu}(t)}{4^{\mu+\nu}(\mu+\nu-1) \Gamma^{2}(\mu+\nu+1)}, \quad \nu=0,1,2, \cdots,
$$

is monotone decreasing as $\nu$ increases. Furthermore

$$
D_{1}(t)=\Lambda_{1}^{2}(t), \quad D_{2}(t)=\frac{8}{t^{2}} \Delta_{2}(t) .
$$

It is known that

$$
\left|\Lambda_{\mu}(t)\right| \leqq \Lambda_{\mu}(0)=1
$$

$t$ real;

hence

$$
Q_{\mu}(t) \rightarrow 0 \quad \text { as } \mu \rightarrow \infty,
$$

and, from (6.7),

$$
Q_{\mu}(t)=2 \sum_{\nu=0}^{\infty} \frac{t^{2(\mu+\nu)} \Lambda_{\mu+\nu}^{2}(t)}{4^{\mu+\nu}(\mu+\nu-1) \Gamma^{2}(\mu+\nu+1)}, \quad \mu>1,
$$

- See S. Minakshisundaram and Otto Szász, On absolute convergence of multiple Fourier series, Trans. Amer. Math. Soc. vol. 61 (1947) pp. 36-53, in particular formula (2.3). 
the series being uniformly convergent. It now follows that

$$
D_{\mu}(t)>0, \quad \text { for } \mu>1, t \text { real; }
$$

hence, from (6.6), we have

$$
\Delta_{\mu-1}(t)>0, \quad \mu>1,
$$

or, in terms of the Bessel functions,

$$
J_{\mu}^{2}(t)-J_{\mu-1}(t) J_{\mu+1}(t)>\frac{1}{\mu+1} J_{\mu}^{2}(t), . \quad \mu>0, t \text { real. }
$$

If, for example, $\mu=3 / 2$, we get

$$
t \sin ^{2} t-3 \cos t(\sin t-t \cos t)>0 \quad \text { for } t>0 \text {. }
$$

We state the main result of this section as:

Theorem 2. If $J_{\mu}(t)$ denotes the Bessel function of index $\mu$, defined by (6.1), then, for $\mu>0$ and all real values of $t$,

$$
J_{\mu}^{2}(t)-J_{\mu-1}(t) J_{\mu+1}(t)>\frac{1}{\mu+1} J_{\mu}^{2}(t) .
$$

Untversity of Cincinnati 